\title{
Associations between CD160 polymorphisms and autoimmune thyroid disease: a case-control study
}

\author{
Weiwei $\mathrm{He}^{1 \dagger}$, Jing Zhao ${ }^{1,2+}$, Xuerong $\mathrm{Liu}^{3}$, Sheli $\mathrm{Li}^{3}$, Kaida $\mathrm{Mu}^{1}$, Jing Zhang ${ }^{1}$ and Jin-an Zhang ${ }^{1 *}$ (D)
}

\begin{abstract}
Background: Recent researches suggest that the CD160/HVEM/LIGHT/BTLA signaling pathway may contribute to the pathogeneses of autoimmune diseases, but the relationship between CD160 polymorphisms and autoimmune thyroid disease (AITD) has not been reported yet. This study aimed to evaluate the associations between CD160 polymorphisms and AITD.
\end{abstract}

Methods: A total of 1017 patients with AITD (634 Graves' disease and 383 Hashimoto's thyroiditis) and 856 unrelated healthy controls were recruited into our study. Odds ratios (ORs) with 95\% confidence interval (95\%Cl) were calculated through logistic regression analyses. The CD160 SNPs were detected using Hi-SNP high-throughput genotyping.

Results: There was a statistically significant difference between Graves' disease patients and the control group with respect to both the genotype distribution $(P=0.014)$ and allele frequency of $r 5744877(P=0.034)$. A significant association of CD160 rs744877 with AITD was observed before adjusted age and gender under a dominant model $(\mathrm{OR}=0.79,95 \% \mathrm{Cl} 0.66-0.95 ; P=0.013)$ and an additive model $(\mathrm{OR}=0.77,95 \% \mathrm{Cl} 0.64-0.94, P=0.008)$, and was also observed after adjusted age and gender under a dominant model $(\mathrm{OR}=0.78,95 \% \mathrm{Cl} 0.65-0.95 ; P=0.011)$ and an additive model $(\mathrm{OR}=0.76,95 \% \mathrm{Cl} 0.63-0.93, P=0.007)$. A significant association of rs 744877 with Graves' disease was observed under an allele model $(\mathrm{OR}=0.84,95 \% \mathrm{Cl} 0.71-0.98, P=0.027)$, a dominant model $(\mathrm{OR}=0.74,95 \% \mathrm{Cl} 0.60$ $0.91 ; P=0.005)$, and an additive model $(\mathrm{OR}=0.72,95 \% \mathrm{Cl} 0.58-0.90, P=0.004)$. Multivariate logistic regression analyses suggested that the association remained significant after adjustment for age and gender. However, rs744877 was not related to Hashimoto's thyroiditis. Furthermore, CD160 rs3766526 was not significantly related to either Graves' disease or Hashimoto's thyroiditis.

Conclusion: This is the first identification of the association of CD160 rs744877 with Graves' disease. Our findings add new data to the genetic contribution to Graves' disease susceptibility and support the crucial role of the CD160/HVEM/LIGHT/BTLA pathway in the pathogenesis of Graves' disease.

Keywords: CD160, Polymorphism, Graves' disease, Hashimoto's thyroiditis

\footnotetext{
* Correspondence: zhangiinan@hotmail.com

'Weiwei He and Jing Zhao contributed equally to this work.

'Department of Endocrinology and Rheumatology, Shanghai University of

Medicine \& Health Sciences Affiliated Zhoupu Hospital, Shanghai 201508,

China

Full list of author information is available at the end of the article
}

(c) The Author(s). 2021 Open Access This article is licensed under a Creative Commons Attribution 4.0 International License, which permits use, sharing, adaptation, distribution and reproduction in any medium or format, as long as you give appropriate credit to the original author(s) and the source, provide a link to the Creative Commons licence, and indicate if changes were made. The images or other third party material in this article are included in the article's Creative Commons licence, unless indicated otherwise in a credit line to the material. If material is not included in the article's Creative Commons licence and your intended use is not permitted by statutory regulation or exceeds the permitted use, you will need to obtain permission directly from the copyright holder. To view a copy of this licence, visit http://creativecommons.org/licenses/by/4.0/. The Creative Commons Public Domain Dedication waiver (http://creativecommons.org/publicdomain/zero/1.0/) applies to the data made available in this article, unless otherwise stated in a credit line to the data. 


\section{Background}

Autoimmune thyroid diseases (AITDs) are endocrine autoimmune diseases with a prevalence of about $5 \%$ worldwide that varies with different racial populations and in areas with different amounts of iodine intake [14]. AITD occurs more frequently in women than in men, and the lifetime risk of Graves' disease (GD) is approximately $3 \%$ for women and $0.5 \%$ for men. Accounting for individuals with undiagnosed and euthyroid Hashimoto's thyroiditis (HT), the true prevalence of HT is estimated to be about $5-10 \%$ of the general population. AITDs encompass several types, including GD, HT, atrophic autoimmune thyroiditis, postpartum thyroiditis, and Graves' ophthalmopathy (GO) [5]. The prevalence of HT is the highest, followed by GD. Because GD and HT are the two main subtypes of AITDs and most published literature investigating the genetics of AITDs have focused on susceptibility genes of either GD or HT, we aimed to explore the susceptibility genes of GD and HT and to include only GD and HT patients in our research $[6,7]$. Though its pathogenesis has not yet been clarified, it has been well accepted that genetic and environmental factors both play crucial roles in the pathogenesis of AITD, of which genetic factors contribute up to $80 \%$ of the risk factors [8-10]. Some studies have confirmed that there is a clear familial predisposition to AITD [11]. GD is characterized by a diffuse toxic goiter caused by thyroid-stimulating hormone (TSH) receptor antibodies (TRAb) and is the most common cause of hyperthyroidism [12]. TRAb binds to TSH receptors on thyroid follicle cells and stimulates the production of thyroid hormones. However, the clinical manifestations of HT are diametrically opposite, with hypothyroidism accompanied by an increase in thyroglobulin antibody (TGAb) and thyroid peroxidase antibody (TPOAb) [10]. HT, also known as chronic lymphocytic thyroiditis or autoimmune thyroiditis, is the most common type of AITD. It is also the most common organ-specific autoimmune disease and the most common cause of hypothyroidism. The pathological feature of HT is the local infiltration of lymphocytes, accompanied by elevated levels of TGAb and TPOAb. Environmental factors affecting the occurrence of HT mainly include high iodine intake, infection, stress, and radiation exposure [7, 13]. There is evidence that many of the susceptibility genes are shared by various autoimmune diseases, such as CTLA4 and PTPN22, which indicate that autoimmune diseases have some similar genetic and molecular pathways [14, 15]. Previous studies have reported that there are many susceptibility genes associated with AITD. Generally, most of those susceptibility genes can be classified into two types. One is thyroid-associated genes including TSHR and TG, and the other is immunity-related genes, such as FOXP3 [16], HLA-DR genes [17], and CD40 [15].
Some other novel AITD genes have also been reported, such as VDR [18, 19] and FCRL3 [20].

Herpesvirus entry mediator (HVEM) is a receptor on immune cells, which is also known as tumor necrosis factor receptor superfamily member 14 (TNFRSF14) [21]. CD160, B- and T-lymphocyte attenuating agents (BTLA), lymphotoxin-like (LIGHT), and lymphotoxinalpha (LTa) are ligands for HVEM [22, 23]. The CD160/ HVEM/LIGHT/BTLA pathway is an essential signaling pathway in regulating immunity, which is involved in the pathogeneses of infection and autoimmunity [2426].. Some gene polymorphisms in the CD160/HVEM/ LIGHT/BTLA pathway have an obvious influence on their functions and are thus associated with host susceptibility to autoimmune diseases [27-31].. The inhibitory co-stimulator CD160, originally named BY55, is an immunoglobulin-like activator of the NK cell receptor and belongs to the glycosylphosphatidylinositol (GPI) fixed membrane protein family. The gene is located at 1q42.3 [32, 33]. There are also several single nucleotide polymorphisms (SNPs) in CD160. A study found that CD160 rs744877 was closely related to the susceptibility of rheumatoid arthritis (RA) and may be involved in the development of RA [34]. rs3766526 of CD160 was identified with data from the 1000 Genomes Project (http:// phase3browser.1000genomes.org/index.html), and it met the selection criteria of MAF more than 0.10 and $r^{2}$ more than 0.8 in the linkage disequilibrium analysis. Currently, there is no study on the relationship between CD160 genetic polymorphisms and AITD, and thus the role of CD160 genetic polymorphisms in the susceptibility to AITD remains unclear. Herein, we report a casecontrol involving a large sample to investigate the relationship between two CD160 SNPs (rs744877 and rs3766526) and AITD in a Chinese Han population.

\section{Methods \\ Study subjects}

The study group consisted of 1017 patients with AITD (634 GD and $383 \mathrm{HT}$ ) and 856 healthy controls. AITD patients were recruited from the Department of Endocrinology, Jinshan Hospital of Fudan University. Both GD and HT are well-characterized autoimmune thyroid disorders with a variety of clinical manifestations. The diagnostic hallmark of GD or HT is the presence of circulating autoantibodies. TGAb and TPOAb can be found in most HT patients. Some HT patients with serious thyroid damage caused by immune system activity and lymphocytic infiltration may have hypothyroidism symptoms, but hypothyroidism is usually absent in a large portion of HT patients and these patients are diagnosed with HT with euthyroidism in clinical practice. HT patients may present with various states of thyroid function, but most will eventually evolve into 
hypothyroidism [13]. Briefly, all AITD patients were diagnosed based on clinical manifestations and laboratory tests. GD patients were diagnosed by the presence of hyperthyroidism, a toxic diffuse goiter, and a positive test for TRAb. HT was mainly diagnosed by TGAb positivity or TPOAb positivity and diffuse hypoechogenicity on thyroid ultrasound, with or without hypothyroidism. AITD patients with other autoimmune diseases as well as genetic diseases were excluded. By asking about their disease history and family history, we excluded those individuals with other autoimmune or genetic diseases and included individuals with AITD alone. Healthy controls were matched by geographic region. Controls with the presence of thyroid disease or other autoimmune disorders and those with severe or chronic inflammatory diseases were excluded. The diagnosis of GO is confirmed by the presence of GD diagnosis and the associated ocular symptoms. This study was approved by the ethics committee of Jinshan Hospital, and written informed consent was obtained from all participants.

\section{Genotyping}

$2 \mathrm{ml}$ of peripheral blood was obtained by venipuncture and collected in an EDTA tube. Extraction of genomic DNA from peripheral blood leukocytes using the standard procedure of the RelaxGene Blood DNA System (Tiangen Biotech, Beijing, China). Moreover, In addition, DNA sample concentrations and A260 / A280 ratios were measured using the Nano Drop 2000 spectrophotometer (Thermo Scientific Company, Waltham, MA, USA) $[35,36]$. The CD160 SNPs were genotyped using a high throughput-SNP (Hi-SNP) genotyping method with technical support from the Shanghai Biowing Applied Biotechnology company, which was based on threeround multiplex PCR coupled with next-generation sequencing. The details of the Hi-SNP genotyping method have been reported in previously published studies [37, 38]. Briefly, DNA was first amplified in a 10ul PCR reaction using the following PCR thermocycling conditions: $95^{\circ} \mathrm{C}$ for $15 \mathrm{~min}(1 \mathrm{cycle}) ; 94{ }^{\circ} \mathrm{C}$ for the $30 \mathrm{~s}, 60^{\circ} \mathrm{C}$ for 10 min and $72{ }^{\circ} \mathrm{C}$ for 30 s ( 4 cycles); $94{ }^{\circ} \mathrm{C}$ for $30 \mathrm{~s}, 60^{\circ} \mathrm{C}$ for $1 \mathrm{~min}$ and $72^{\circ} \mathrm{C}$ for 30 s ( 20 cycles). PCR products in the first round were then amplified for the second PCR, and PCR products from the second round were further amplified under PCR thermocycling conditions used in previous studies $[37,38]$. The SNPs were directly identified by mapping targeted sequences to the SNP reference sequences (NCBI, dbSNP build 142) using BWA (v0.7.12) and samtools (v0.1.19). The forward and reverse primers in the amplification for rs744877 were 5 '-GCTGACCT TCCAGCTCCTCTG-3' and 5'-CTGGACCTCACTCA GCCTCAC-3, respectively. The forward and reverse primers in the amplification for rs3766526 were 5'-
ATATACTCAATCTGGCCATCAGC-3' and 5'-TTTT CAATGGGTAGGAGGTAAGAC-3', respectively.

\section{Statistical analysis}

STATA was used for the statistical analysis. HardyWeinberg equilibrium (HWE) was evaluated using the X2 test. A Chi-square test was used to compare allele and genotype frequencies between cases and controls. Through the dominant model, allelic model, homozygous model, recessive model, and additive model, the relationship between CD160 and AITD including GD and HT was analyzed. Multiple logistic regression analysis was also performed, and age and gender were used as confounding factors. $\mathrm{P}<0.05$ was considered significant. Before starting our research, we had done a power analysis to calculate the sample size with an online power and sample size calculation software (http://www. openepi.com/Menu/OE_Menu.htm). We calculated the power for rs3766526 because the rs3766526 MAF of Chinese in 1000 Genomes Project was lower than that of rs744877 (18.5\% vs $30.3 \%)$ and a larger sample size was needed to find a statistically significant association. When the expected OR defined as 1.35 , a sufficient power of over $80 \%$ required a combined set of 530 cases and 530 controls. Moreover, 1017 AITD cases and 856 controls in our study had a power of $99.6 \%$ in discovering a significant difference between AITD cases and controls. Six hundred thirty-four GD cases and 856 controls had a power of $98.7 \%$ in finding an obvious difference between GD cases and controls. six hundred thirty-four HT cases and 856 controls had a power of $94.8 \%$ in finding an obvious difference between HT cases and controls. Therefore, the sample size in our study was suitably powered.

\section{Results \\ Subject features}

The baseline data for this study is shown in Table 1 . There were 634, 383, and 856 individuals in the GD, HT, and control groups, respectively. In the GD group, 446 (70.35\%) individuals were females and 188 (29.65\%) were males. Among them, 135 (21.29\%) individuals had a positive family history of AITD and 100 (15.77\%) had ophthalmopathy. There were 171 (26.97\%) patients with an I degree goiter, 293 (46.21\%) patients with a II degree goiter, and $43(6.79 \%)$ patients with a III degree goiter. In the HT group, 328 (85.64\%) individuals were females and 55 (14.36\%) were males. 61 (15.93\%) individuals in the HT group had a positive family history of AITD. Similarly, there were $92(24.02 \%)$ patients with an I degree goiter, 128 (33.42\%) patients with a II degree goiter, and $8(2.09 \%)$ patients with a III degree goiter in the HT group. The average age in the GD group and HT group was $41.57 \pm 14.47$ years and $41.59 \pm 14.43$ years, 
Table 1 Baseline clinical and demographics features of the study patients

\begin{tabular}{|c|c|c|c|}
\hline Items & GD & HT & Controls \\
\hline Number & 634 & 383 & 856 \\
\hline \multicolumn{4}{|l|}{ Gender } \\
\hline Male & 188 (29.65\%) & 55 (14.36\%) & 357 (41.71\%) \\
\hline Female & 446 (70.35\%) & $328(85.64 \%)$ & 499 (58.29\%) \\
\hline Age (years) & $41.57 \pm 14.47$ & $41.59 \pm 14.43$ & $39.30 \pm 9.71$ \\
\hline \multicolumn{4}{|l|}{ Goiter } \\
\hline No goiter & 127 (20.03\%) & 155 (40.47\%) & - \\
\hline Degree I & 171 (26.97\%) & 92 (24.02\%) & - \\
\hline Degree II & 293 (46.21\%) & 128 (33.42\%) & - \\
\hline Degree III & 43 (6.79\%) & 8 (2.09\%) & - \\
\hline \multicolumn{4}{|c|}{ Family history } \\
\hline$(+)$ & 135 (21.29\%) & 61 (15.93\%) & - \\
\hline$(-)$ & 499 (78.71\%) & 322 (84.07\%) & - \\
\hline \multicolumn{4}{|c|}{ Ophthalmopathy } \\
\hline$(+)$ & 100 (15.77\%) & $3(0.78 \%)$ & - \\
\hline$(-)$ & 534 (84.23\%) & 380 (99.22\%) & - \\
\hline
\end{tabular}

GD Graves' disease, HT Hashimoto's thyroiditis

respectively. Among the 856 healthy controls, 499 (58.29\%) individuals were females and 357 (41.71\%) were males. Age and gender were different between patient and control groups $(P<0.05)$. No deviation from the HWE was observed for both rs744877 and rs3766526 in the controls $(P>0.05)$.

\section{Association between rs744877 and AITD}

The genotyping results of all patients and controls are shown in Table 2. In AITD subjects, the genotype frequencies of $\mathrm{AA}, \mathrm{AC}$, and $\mathrm{CC}$ in rs744877 polymorphism were $47.89,41.79$, and $10.32 \%$, respectively, whereas those in the control group were 42.17, 47.66, and $10.17 \%$, respectively (Table 2 ). In GD subjects, the genotype frequencies of $\mathrm{AA}, \mathrm{AC}$, and $\mathrm{CC}$ in the rs744877 polymorphism were 49.53, 40.54, and 9.93\%, respectively. In HT subjects, the genotype frequencies of AA, $\mathrm{AC}$, and $\mathrm{CC}$ in the rs744877 locus were 45.17, 43.86, and $10.97 \%$, respectively. Our result showed that there was a statistically significant difference between AITD patients and the control group with respect to the genotype distribution of rs744877 $(P=0.031)$. Obvious differences for both the genotype distribution $(P=0.014)$ and allele frequencies of $\operatorname{rs744877}(P=0.034)$ were observed between GD patients and controls. However, there was no difference between HT patients and the control group with respect to both the genotype distribution $(P=0.464)$ and allele frequencies of rs744877 $(P=0.593)$.

For rs744877, the allele model was for $\mathrm{C}$ vs $\mathrm{A}$, the dominant model was for $\mathrm{CC} / \mathrm{AC}$ vs $\mathrm{AA}$, the recessive model was for $\mathrm{CC}$ vs $\mathrm{AA} / \mathrm{AC}$, the homozygous model was for $\mathrm{CC}$ vs AA, and the additive model was for AC vs AA. As shown in Table 3, a significant association of CD160 rs744877 with AITD was observed under both the dominant model $(\mathrm{OR}=0.79,95 \% \mathrm{CI} 0.66-0.95, P=$ $0.013)$ and additive model $(\mathrm{OR}=0.77,95 \% \mathrm{CI} 0.64-0.94$; $P=0.008)$. Multivariate logistic regression analysis suggested that the association remained significant after adjusting for age and gender (Table 3 ).

As shown in Table 4, a significant association of CD160 rs744877 with GD was observed under the allele model $(\mathrm{OR}=0.84$, 95\%CI $0.71-0.98, P=0.027)$, dominant model $(\mathrm{OR}=0.74,95 \% \mathrm{CI} 0.60-0.91 ; P=0.005)$, and additive model $(\mathrm{OR}=0.72$, 95\% CI $0.58-0.90, P=0.004)$, Multivariate logistic regression analyses suggested that

Table 2 Allele frequencies and genotype distribution of CD160 polymorphisms in AITD patients and controls

\begin{tabular}{|c|c|c|c|c|c|c|c|}
\hline $\begin{array}{l}\text { Gene/SNP } \\
\text { CD160 }\end{array}$ & $\begin{array}{l}\text { Controls } \\
\text { n (\%) }\end{array}$ & $\begin{array}{l}\text { AITD } \\
\text { n (\%) }\end{array}$ & $\begin{array}{l}P \text { value } \\
\text { AITD vs. Control }\end{array}$ & $\begin{array}{l}\text { GD } \\
\text { n (\%) }\end{array}$ & $\begin{array}{l}P \text {-Value } \\
\text { GD vs. Control }\end{array}$ & $\begin{array}{l}\text { HT } \\
\text { n (\%) }\end{array}$ & $\begin{array}{l}P \text { value } \\
\text { HT vs. Control }\end{array}$ \\
\hline \multicolumn{8}{|l|}{ rs744877 } \\
\hline$A$ & 1130 (66\%) & 1399 (68.78\%) & 0.071 & 885 (69.69\%) & 0.034 & $514(67.10 \%)$ & 0.593 \\
\hline$C$ & $582(34 \%)$ & 635 (31.22\%) & & 385 (30.31\%) & & 252 (32.90\%) & \\
\hline AA & 361 (42.17\%) & 487 (47.89\%) & 0.031 & 314 (49.53\%) & 0.014 & 173 (45.17\%) & 0.464 \\
\hline$A C$ & $408(47.66 \%)$ & 425 (41.79\%) & & 257 (40.54\%) & & 168 (43.86\%) & \\
\hline$C C$ & 87 (10.17\%) & 105 (10.32\%) & & 63 (9.93\%) & & 42 (10.97\%) & \\
\hline \multicolumn{8}{|l|}{ rs3766526 } \\
\hline G & 1406 (82.13\%) & $1636(80.43 \%)$ & 0.186 & 1013 (79.89\%) & 0.123 & $623(81.33 \%)$ & 0.635 \\
\hline A & 306 (17.87\%) & 398 (19.57\%) & & 255 (20.11\%) & & 143 (18.67\%) & \\
\hline GG & $574(67.06 \%)$ & 659 (64.80\%) & 0.319 & 407 (64.20\%) & 0.187 & $252(65.80 \%)$ & 0.888 \\
\hline $\mathrm{GA}$ & 258 (30.14\%) & 318 (31.27\%) & & 199 (31.39\%) & & 119 (31.07\%) & \\
\hline $\mathrm{AA}$ & $24(2.80 \%)$ & 40 (3.93\%) & & $28(4.41 \%)$ & & $12(3.13 \%)$ & \\
\hline
\end{tabular}


Table 3 Associations of two polymorphisms in the CD160 gene with AITD before and after adjusting for confounders

\begin{tabular}{|c|c|c|c|c|}
\hline \multirow[t]{2}{*}{ Comparison models } & \multicolumn{2}{|c|}{ Unadjusted estimates } & \multicolumn{2}{|c|}{ Adjusted estimates $^{a}$} \\
\hline & OR $(95 \% \mathrm{Cl})$ & $P$ values & OR $(95 \% \mathrm{Cl})$ & $P$ values \\
\hline \multicolumn{5}{|l|}{ rs744877 } \\
\hline Allele Model & $0.88(0.77-1.01)$ & 0.069 & $0.88(0.76-1.01)$ & 0.066 \\
\hline Dominant Model & $0.79(0.66-0.95)$ & 0.013 & $0.78(0.65-0.95)$ & 0.011 \\
\hline Recessive model & $1.02(0.75-1.37)$ & 0.909 & $1.03(0.76-1.40)$ & 0.864 \\
\hline Homozygous model & $0.89(0.65-1.23)$ & 0.489 & $0.90(0.65-1.24)$ & 0.528 \\
\hline Additive model & $0.77(0.64-0.94)$ & 0.008 & $0.76(0.63-0.93)$ & 0.007 \\
\hline \multicolumn{5}{|l|}{ rs3766526 } \\
\hline Allele Model & $1.12(0.95-1.32)$ & 0.185 & $1.12(0.94-1.32)$ & 0.197 \\
\hline Dominant Model & $1.11(0.91-1.34)$ & 0.305 & $1.10(0.90-1.34)$ & 0.354 \\
\hline Recessive model & $1.42(0.85-2.37)$ & 0.182 & $1.49(0.88-2.52)$ & 0.138 \\
\hline Homozygous model & $1.45(0.86-2.44)$ & 0.159 & $1.51(0.89-2.57)$ & 0.126 \\
\hline Additive model & $1.07(0.88-1.31)$ & 0.484 & $1.06(0.86-1.30)$ & 0.579 \\
\hline
\end{tabular}

AITD autoimmune diseases, OR Odds ratio, $95 \% \mathrm{Cl} 95 \%$ confidence interval; ${ }^{\text {a }}$ Age and gender were adjusted in the multivariate logistic regression analyses)

the association was significant after adjusting for age and gender (Table 4).

As shown in Table 5, no significant association of CD160 rs744877 with HT was observed under all models (Table 5, $P>0.05$ ). Besides, no association of rs744877 with HT was observed after adjustment for age and gender (Table 5).

Subgroup analysis of patients with GD ophthalmopathy showed a significant difference in the genotype distribution of rs744877 between patients without ophthalmopathy and controls $(P=0.018$; Supplementary Table 1). Subgroup analysis by gender revealed that rs744877 was significantly associated with GD in women, but minimally associated with GD in men, in terms of genotype distribution $(P<0.001)$ and allele frequency $(P=0.004)$ (Supplementary Tables 2 and 3$)$. The results of subgroup analyses stratified by family history and degree of goiter are presented in the accompanying tables (Supplementary Tables 4, 5, 6 and 7).

\section{Association between rs3766526 and AITD}

As shown in Table 2, there was no difference between the patient group and the control group with respect to both the genotype distribution $(P=0.319)$ and allele frequency of CD160 rs3766526 $(P=0.186)$. No difference for both the genotype distribution $(P=0.187)$ and allele frequencies of rs3766526 $(P=0.123)$ was observed between GD patients and controls. There was no significant finding between HT cases and controls in both the genotype distribution $(P=0.888)$ and allele frequencies of rs3766526 $(P=0.635)$.

Table 4 Associations of two polymorphism models in the CD160 gene with GD before and after adjusting for confounders

\begin{tabular}{|c|c|c|c|c|}
\hline \multirow[t]{2}{*}{ Comparison models } & \multicolumn{2}{|c|}{ Unadjusted estimates } & \multicolumn{2}{|c|}{ Adjusted estimates $^{\mathrm{a}}$} \\
\hline & OR $(95 \% \mathrm{Cl})$ & $P$ values & OR $(95 \% \mathrm{Cl})$ & $P$ values \\
\hline \multicolumn{5}{|l|}{ rs744877 } \\
\hline Allele Model & $0.84(0.71-0.98)$ & 0.027 & $0.84(0.71-0.98)$ & 0.030 \\
\hline Dominant Model & $0.74(0.60-0.91)$ & 0.005 & $0.74(0.60-0.92)$ & 0.005 \\
\hline Recessive model & $0.98(0.69-1.37)$ & 0.886 & $0.98(0.70-1.39)$ & 0.923 \\
\hline Homozygous model & $0.83(0.58-1.19)$ & 0.315 & $0.84(0.58-1.20)$ & 0.339 \\
\hline Additive model & $0.72(0.58-0.90)$ & 0.004 & $0.72(0.58-0.90)$ & 0.004 \\
\hline \multicolumn{5}{|l|}{ rs3766526 } \\
\hline Allele Model & $1.16(0.96-1.39)$ & 0.122 & $1.14(0.95-1.38)$ & 0.160 \\
\hline Dominant Model & $1.14(0.91-1.41)$ & 0.250 & $1.12(0.90-1.40)$ & 0.295 \\
\hline Recessive model & $1.60(0.92-2.79)$ & 0.096 & $1.54(0.88-2.70)$ & 0.130 \\
\hline Homozygous model & $1.65(0.94-2.88)$ & 0.081 & $1.57(0.89-2.77)$ & 0.116 \\
\hline Additive model & $1.09(0.87-1.36)$ & 0.462 & $1.08(0.86-1.36)$ & 0.502 \\
\hline
\end{tabular}


Table 5 Associations of two polymorphism models in the CD160 gene with HT before and after adjusting for confounders

\begin{tabular}{|c|c|c|c|c|}
\hline \multirow[t]{2}{*}{ Comparison models } & \multicolumn{2}{|c|}{ Unadjusted estimates } & \multicolumn{2}{|c|}{ Adjusted estimates $^{a}$} \\
\hline & OR $(95 \% \mathrm{Cl})$ & $P$ values & OR $(95 \% \mathrm{Cl})$ & $P$ values \\
\hline \multicolumn{5}{|l|}{ rs744877 } \\
\hline Allele Model & $0.95(0.79-1.14)$ & 0.585 & $0.95(0.79-1.16)$ & 0.632 \\
\hline Dominant Model & $1.06(0.82-1.37)$ & 0.664 & $1.07(0.82-1.40)$ & 0.615 \\
\hline Recessive model & $1.09(0.74-1.61)$ & 0.669 & $1.11(0.73-1.67)$ & 0.629 \\
\hline Homozygous model & $1.01(0.67-1.52)$ & 0.972 & $1.04(0.68-1.60)$ & 0.860 \\
\hline Additive model & $0.86(0.67-1.11)$ & 0.244 & $0.86(0.66-1.12)$ & 0.262 \\
\hline \multicolumn{5}{|l|}{ rs3766526 } \\
\hline Allele Model & $1.06(0.85-1.32)$ & 0.631 & $1.09(0.86-1.39)$ & 0.457 \\
\hline Dominant Model & $0.89(0.69-1.13)$ & 0.325 & $0.89(0.69-1.14)$ & 0.354 \\
\hline Recessive model & $1.12(0.55-2.27)$ & 0.750 & $1.46(0.69-3.09)$ & 0.322 \\
\hline Homozygous model & $1.14(0.56-2.31)$ & 0.719 & $1.45(0.69-3.08)$ & 0.328 \\
\hline Additive model & $1.05(0.81-1.37)$ & 0.713 & $1.04(0.79-1.37)$ & 0.785 \\
\hline
\end{tabular}

HT Hashimoto's thyroiditis, OR Odds ratio, $95 \% \mathrm{Cl} 95 \%$ confidence interval; ${ }^{a}$ Age and gender were adjusted in the multivariate logistic regression analyses

For rs3766526, the allele model was for A vs G, the dominant model was for AA/GA vs GG, the recessive model was for AA vs GG/GA, the homozygous model was for AA vs GG, and the additive model was for GA vs GG. As shown in Table 3, no significant association of CD160 rs3766526 with AITD was observed under all comparison models (Table 3, $P>0.05$ ). Multivariate logistic regression analyses suggested that the association was not yet significant after adjustment for age and gender (Table 3). CD160 rs3766526 was also not related to either GD or HT under all comparison models (Tables 4 and 5, $P>0.05$ ).

Although neither genotype distribution nor allele frequency of rs3766526 differed between GD patients and controls (Table 2), it is noteworthy that the allele frequency of rs3766526 differed significantly between GO patients and controls $(P=0.039$; Supplementary Table 1$)$. Subgroup analysis by sex revealed that rs3766526 was not associated with GD and HT in females, but was significantly associated with HT in males, both in terms of genotype distribution $(P<0.001)$ and allele frequency $(P=$ 0.015) (Supplementary Table 3), suggesting that rs3766526 may be associated with the onset of HT. CD160 rs3766526 is a variant in $3^{\prime}$-UTR which often contains regulatory regions for post-transcriptional modification. Therefore, rs3766526 may have an impact on the expression of CD160 by influencing post-transcriptional modification, and thus a change in the function of the CD160/HVEM/LIGHT/BTLA pathway. Outcomes of the subgroup analyses stratified by family history and goiter degree for rs3766526 are shown in the supplementary tables (Supplementary Tables 4, 5, 6 and 7).

\section{Discussion}

In this study, we explored two SNPs in CD160 and found an obvious relationship between CD160 rs744877 and GD. This correlation was also confirmed through multivariate logistic regression analysis. However, our study did not find any correlation between rs744877 and HT. To our knowledge, it is the first study on the linkage of CD160 polymorphisms to AITD.

The CD160/HVEM/LIGHT/BTLA pathway is an essential signaling pathway in regulating immunity, which is involved in the pathogeneses of infection and autoimmunity [22, 23, 39]. CD160, BTLA, LIGHT, and LTa all are ligands for HVEM, but they exert different regulatory roles [22, 23]. The binding of LIGHT or LTa to HVEM stimulates costimulatory signals, but the binding of BTLA or CD160 to HVEM initiates co-suppression signals, and thus the CD160/HVEM/LIGHT/BTLA costimulatory/ co-suppression pathway is a bidirectional switch that adjusts $\mathrm{T}$ cell activation and takes an important part in regulating immune responses [24, 40, 41]. Although the binding of LIGHT or LTa to HVEM promotes $\mathrm{T}$ cell activation, HVEM predominantly shows negative regulatory effects mediated by BTLA and CD160 [22, 39]. The cysteine concentration 1 (CRD1) region in HVEM molecule is responsible for the combination of CD160 and BTLA, and the deletion of HVEM CRD1 region could block the combination of CD160/ BTLA and HVEM and eliminate the inhibitory effect. Thus, HVEM can be transformed into a dominant costimulator and enhance the immune response, even causing graft rejection [42]. Some observational studies have revealed that these genes are aberrantly expressed in patients with autoimmune diseases [43-45]. In addition, animals with knock-out of those members in the CD160/HVEM/LIGHT/BTLA signaling pathway could change the development or progression of autoimmune diseases, such as colitis and atopic dermatitis $[46,47]$. 
Recent genetic studies have identified an association of CD160/HVEM/LIGHT/BTLA pathway-related genes with autoimmune diseases [48]. HVEM (TNFRSF14) rs6684865 was related to primary sclerosing cholangitis (PSC), RA, and multiple sclerosis (MS) [30, 48, 49]. The findings from our study prove that CD160 rs744877 is related to GD, which adds new evidence for the roles of the CD160/HVEM/LIGHT/BTLA pathway in autoimmune diseases. CD160 is an essential member of the CD160/HVEM/LIGHT/BTLA pathway, and its role in immunity has been widely recognized [50]. CD160 is highly expressed in the spleen, small intestine, and peripheral blood, but is lowly expressed in the brain, liver, heart, thymus, and other tissues [25, 32, 33]. In the immune system, CD160 is a costimulatory receptor molecule and is expressed on multiple immune cells, including intestinal intraepithelial $\mathrm{T}$ lymphocytes, CD56dimCD16+ NK lymphocytes, and a minor subset of CD4+ T cells and CD8+ T cells [51].. In the pathogeneses of GD, antigen-presenting cells (APC), T cells, and $\mathrm{B}$ cells are all involved, and the dysfunction of one type of immune cells can further lead to aberrant function of other immune cells [10]. CD160 primarily functioning as a costimulatory receptor molecule. CD160 is an essential member of the CD160/HVEM/LIGHT/ BTLA pathway, and its role in immunity has been widely recognized. Therefore, CD160 plays an important role in the maintenance of immune tolerance and the prevention of autoimmune diseases. More importantly, one of our recent study suggested that CD160 was differentially expressed on the surface of $\mathrm{CD}^{+} \mathrm{T}$ cells between GD patients and healthy controls $(P=0.002)$, which suggested that CD160 dysfunction was involved in the pathogenesis of GD [52]. CD160 SNP may lead to impairment of its immunomodulatory function by affecting its expression or function. Therefore, aberrant expression or function of CD160 can first result in the dysfunction of $\mathrm{T}$ cells, which may further cause the activation of antibody-producing B lymphocytes and lead to the development of GD. CD160 has a higher affinity for HVEM and can inhibit CD4 $4^{+} \mathrm{T}$ cell proliferation and cytokine secretion after cross-linking [51]. Therefore, CD160 plays an important role in the maintenance of immune tolerance and the prevention of autoimmune diseases $[25,53]$.

Though our study indicated CD160 rs744877 locus is a susceptible factor for GD, the molecular mechanisms underlying the roles of CD160 rs744877 in autoimmune diseases are still unclear. CD160 rs744877 is an intronic SNP and is unable to act by changing the composition of amino acids or the protein structure of CD160. One possibility is that CD160 rs744877 takes part in disease development by impacting its expression or posttranslational modification. CD160 polymorphisms may not be related to HT due to the different molecular mechanisms between GD and HT. Though both GD and HT are similar autoimmune diseases occurring in the same organ, they still may have some distinct molecular mechanisms [8, 54]. Previous studies also suggested several genes are differently associated with GD and HT, such as BTG1 and TSHR. Therefore, the associations of CD160 polymorphisms with GD and HT may be different, as CD160 may exert different roles in the pathogeneses of GD and HT. Another explanation was the limited number of HT patients in our study, which may cause low statistical power to detect a statistically significant relationship. Finally, subgroup analyses by gender found that rs3766526 was not associated with GD and HT in females, but it was significantly associated with HT in males for both the genotype distribution $(P<0.001)$ and allele frequencies $(P=0.015)$ (Supplementary Table 3 ), which suggested that the genderspecific association of rs3766526 with HT was possible. However, the finding above may be biased by the limited sample size in the subgroup analyses by gender, and studies with larger sample sizes are recommended to provide a more precise evaluation of the gender-specific association of rs3766526 with HT. Similar to other published gene-association studies, our study did not perform a replication study, which was due to the limited number of AITD DNA samples in our clinical biobank of AITD. To ensure the reliability of the findings in our study, we attempted to collect more AITD samples, and the sample size in our study is relatively large compared with other published case-control studies on the susceptibility genes of AITD. Moreover, the sample size calculation also suggested that the sample size in our study was large enough to assess associations adequately. Our study focused on the relationship between AITD and controls, and from this perspective the number of patients was sufficient to assess the relationship between CD160 SNPs and AITD. However, when subgroup analysis was done, there were fewer HT patients, which may lead to low statistical power in assessing the relationship between CD160 SNPs and HT. Nevertheless, the findings in our study still need to be validated in future studies, especially for those from other countries or populations.

\section{Conclusion}

In conclusion, this is the first demonstration of the association of CD160 rs744877 with GD and supports the critical role of the CD160 / HVEM / LIGHT / BTLA pathway in the pathogenesis of GD.

\section{Abbreviations}

AITDs: Autoimmune thyroid diseases; GD: Graves' disease; HT: Hashimoto's thyroiditis; GO: Graves' ophthalmopathy; TSH: Thyroid-stimulating hormone receptor antibodies (TRAb); TRAb: Thyroid-stimulating hormone receptor 
antibodies; TGAb: Thyroglobulin antibody; TPOAb: Thyroid peroxidase antibody; HVEM: Herpesvirus entry mediator; TNFRSF14: Tumor necrosis factor receptor superfamily member 14; BTLA: B- and T-lymphocyte attenuating agents; SNPs: Single nucleotide polymorphisms; RA: Rheumatoid arthritis; Hi-SNP: High throughput-SNP; PSC: Primary sclerosing cholangitis; MS: Multiple sclerosis; APC: Antigen-presenting cells

\section{Supplementary Information}

The online version contains supplementary material available at https://doi. org/10.1186/s12902-021-00810-w.

Additional file 1: Supplementary Table 1. Allele frequencies and genotype distribution of CD160 polymorphisms between GD patients and controls in the subgroup analysis by ophthalmopathy.

Supplementary Table 2. Allele frequencies and genotype distribution of CD160 polymorphisms between AITD patients and controls in females. Supplementary Table 3. Allele frequencies and genotype distribution of CD160 polymorphisms between AITD patients and controls in males. Supplementary Table 4. Allele frequencies and genotype distribution of CD160 polymorphisms between AITD patients and controls stratified by family history. Supplementary Table $\mathbf{5}$. Allele frequencies and genotype distribution of CD160 polymorphisms between GD patients and controls stratified by family history. Supplementary Table 6 . Allele frequencies and genotype distribution of CD160 polymorphisms between HT patients and controls stratified by family history. Supplementary Table 7. Allele frequencies and genotype distribution of CD160 polymorphisms between AITD patients and controls stratified by goiter.

\section{Acknowledgments}

We are grateful to all the individuals who participated in this study.

\section{Authors' contributions}

All of the authors contributed to the design of the study. JA-Z designed the study. $\mathrm{WH}$ and $J Z$ wrote the manuscript. XR L collected, analyzed the data with SL L, KD M and JZ. WY gave much advice and directions in both study design and preparing of the manuscript. All the authors have read and approved the final submitted version. Weiwei He and Jing Zhao these authors are contributed to the article equally.

\section{Funding}

The present work was supported by grants from the National Natural Science Foundation of China (No. 81670722, 81900710), Shanghai Natural Science Foundation (No.18ZR1433800); Health Scientific Research Project of Pudong New District Health Commission (No. PW2019D-12) and The Project of Shanghai Medical Key Specialty (No. ZK2019C09). The funding body plays no role in the design of the study and collection, analysis, and interpretation of data and in writing the manuscript.

\section{Availability of data and materials}

Data sharing is not applicable to this article, as no datasets were generated or analyzed during the current study.

\section{Declarations}

\section{Ethics approval and consent to participate}

The study was approved by the research Ethics Committee at the Jinshan Hospital of Fudan University, and written informed consent was obtained from all participants.

\section{Consent for publication}

Not applicable.

\section{Competing interests}

The authors declare that they have no competing interests.

\section{Author details}

${ }^{1}$ Department of Endocrinology and Rheumatology, Shanghai University of Medicine \& Health Sciences Affiliated Zhoupu Hospital, Shanghai 201508,
China. ${ }^{2}$ Department of Endocrinology, Nanjing Medical University Affiliated Wuxi People's Hospital, Wuxi 214000, China. ${ }^{3}$ Department of Endocrinology, Affiliated Hospital of Yanan University, Yan'an, Shanxi, China.

Received: 13 November 2020 Accepted: 28 June 2021

Published online: 08 July 2021

\section{References}

1. Valdes S, Maldonado-Araque C, Lago-Sampedro A, Lillo JA, Garcia-Fuentes E, Perez-Valero V, et al. Population-based national prevalence of thyroid dysfunction in Spain and associated factors: Di@bet.es study. Thyroid. 2017: 27(2):156-66.

2. Khattak RM, Ittermann $T$, Nauck $M$, Below $H$, Volzke $H$. Monitoring the prevalence of thyroid disorders in the adult population of Northeast Germany. Popul Health Metrics. 2016:14:39.

3. Shan Z, Chen L, Lian X, Liu C, Shi B, Shi L, et al. lodine status and prevalence of thyroid disorders after introduction of mandatory universal salt iodization for 16 years in China: a cross-sectional study in 10 cities. Thyroid. 2016;26(8):1125-30.

4. McLeod DS, Cooper DS, Ladenson PW, Whiteman DC, Jordan SJ. Race/ ethnicity and the prevalence of thyrotoxicosis in young Americans. Thyroid. 2015;25(6):621-8.

5. Hadj-Kacem H, Rebuffat S, Mnif-Feki M, Belguith-Maalej S, Ayadi H, PeraldiRoux S. Autoimmune thyroid diseases: genetic susceptibility of thyroidspecific genes and thyroid autoantigens contributions. Int J Immunogenet. 2009;36(2):85-96.

6. Fujii A, Inoue N, Watanabe M, Kawakami C, Hidaka Y, Hayashizaki $Y$, et al. TSHR gene polymorphisms in the enhancer regions are most strongly associated with the development of Graves' disease, especially intractable disease, and of Hashimoto's disease. Thyroid. 2017;27(1):111-9.

7. Antonelli A, Ferrari SM, Corrado A, Di Domenicantonio A, Fallahi P. Autoimmune thyroid disorders. Autoimmun Rev. 2015;14(2):174-80.

8. Tomer $Y$. Mechanisms of autoimmune thyroid diseases: from genetics to epigenetics. Annu Rev Pathol. 2014;9:147-56.

9. Ajjan RA, Weetman AP. The pathogenesis of Hashimoto's thyroiditis: further developments in our understanding. Horm Metab Res. 2015;47(10):702-10.

10. Lee HJ, Li CW, Hammerstad SS, Stefan M, Tomer Y. Immunogenetics of autoimmune thyroid diseases: a comprehensive review. J Autoimmun. 2015. 64:82-90.

11. Brix TH, Kyvik KO, Christensen $K$, Hegedus L. Evidence for a major role of heredity in Graves' disease: a population-based study of two Danish twin cohorts. J Clin Endocrinol Metab. 2001;86(2):930-4.

12. Burch HB, Cooper DS. Management of Graves disease: a review. Jama. 2015; 314(23):2544-54.

13. Caturegli P, De Remigis A, Rose NR. Hashimoto thyroiditis: clinical and diagnostic criteria. Autoimmun Rev. 2014;13(4-5):391-7.

14. Heward JM, Brand OJ, Barrett JC, Carr-Smith JD, Franklyn JA, Gough SC. Association of PTPN22 haplotypes with Graves' disease. J Clin Endocrinol Metab. 2007;92(2):685-90.

15. Yang J, Qin Q, Yan N, Zhu YF, Li C, Yang XJ, et al. CD40 C/T(-1) and CTLA-4 $\mathrm{a} / \mathrm{G}$ (49) SNPs are associated with autoimmune thyroid diseases in the Chinese population. Endocrine. 2012;41(1):111-5.

16. Shehjar F, Afroze D, Misgar RA, Malik SA, Laway BA. Association of FoxP3 promoter polymorphisms with the risk of Graves' disease in ethnic Kashmiri population. Gene. 2018;672:88-92.

17. Yanagawa T, Mangklabruks A, Chang YB, Okamoto Y, Fisfalen ME, Curran PG, et al. Human histocompatibility leukocyte antigen-DQA ${ }^{*} 0501$ allele associated with genetic susceptibility to Graves' disease in a Caucasian population. J Clin Endocrinol Metab. 1993;76(6):1569-74.

18. Zarrin R, Bagheri M, Mehdizadeh A, Ayremlou P, Faghfouri AH. The association of Fokl and Apal polymorphisms in vitamin D receptor gene with autoimmune thyroid diseases in the northwest of Iran. Med J Islam Repub Iran. 2018;32:4.

19. Gao XR, Yu YG. Meta-analysis of the association between vitamin D receptor polymorphisms and the risk of autoimmune thyroid disease. Int J Endocrinol. 2018;2018:2846943.

20. Simmonds MJ, Brand OJ, Barrett JC, Newby PR, Franklyn JA, Gough SC. Association of $\mathrm{fc}$ receptor-like 5 (FCRL5) with Graves' disease is secondary to the effect of FCRL3. Clin Endocrinol. 2010;73(5):654-60.

21. Bechill J, Muller WJ. Herpesvirus entry mediator (HVEM) attenuates signals mediated by the lymphotoxin beta receptor (LTbetaR) in human cells stimulated by the shared ligand LIGHT. Mol Immunol. 2014;62(1):96-103. 
22. Murphy TL, Murphy KM. Slow down and survive: enigmatic immunoregulation by BTLA and HVEM. Annu Rev Immunol. 2010;28:389411.

23. Shui JW, Steinberg MW, Kronenberg M. Regulation of inflammation, autoimmunity, and infection immunity by HVEM-BTLA signaling. J Leukoc Biol. 2011;89(4):517-23.

24. Cai G, Freeman GJ. The CD160, BTLA, LIGHT/HVEM pathway: a bidirectiona switch regulating T-cell activation. Immunol Rev. 2009;229(1):244-58.

25. del Rio ML, Lucas CL, Buhler L, Rayat G, Rodriguez-Barbosa J. HVEM/LIGHT/ BTLA/CD160 cosignaling pathways as targets for immune regulation. J Leukoc Biol. 2010;87(2):223-35.

26. Jones A, Bourque J, Kuehm L, Opejin A, Teague RM, Gross C, et al. Immunomodulatory functions of BTLA and HVEM govern induction of extrathymic regulatory $T$ cells and tolerance by dendritic cells. Immunity. 2016:45(5):1066-77.

27. Ananthakrishnan AN, Oxford EC, Nguyen DD, Sauk J, Yajnik V, Xavier RJ. Genetic risk factors for Clostridium difficile infection in ulcerative colitis. Aliment Pharmacol Ther. 2013;38(5):522-30.

28. Lopez Herraez D, Martinez-Bueno M, Riba L, Garcia de la Torre I, Sacnun M, Goni M, et al. Rheumatoid arthritis in Latin Americans enriched for Amerindian ancestry is associated with loci in chromosomes 1, 12, and 13, and the HLA class II region. Arthritis Rheum. 2013;65(6):1457-67.

29. Kurreeman FA, Stahl EA, Okada Y, Liao K, Diogo D, Raychaudhuri S, et al. Use of a multiethnic approach to identify rheumatoid- arthritis-susceptibility loci, 1 p36 and 17q12. Am J Hum Genet. 2012;90(3):524-32.

30. Folseraas T, Melum E, Rausch P, Juran BD, Ellinghaus E, Shiryaev A, et al. Extended analysis of a genome-wide association study in primary sclerosing cholangitis detects multiple novel risk loci. J Hepatol. 2012;57(2):366-75.

31. Dubois PC, Trynka G, Franke L, Hunt KA, Romanos J, Curtotti A, et al. Multiple common variants for celiac disease influencing immune gene expression. Nat Genet. 2010;42(4):295-302

32. Maiza H, Leca G, Mansur IG, Schiavon V, Boumsell L, Bensussan A. A novel 80-kD cell surface structure identifies human circulating lymphocytes with natural killer activity. J Exp Med. 1993;178(3):1121-6.

33. Anumanthan A, Bensussan A, Boumsell L, Christ AD, Blumberg RS, Voss SD, et al. Cloning of BY55, a novel lg superfamily member expressed on NK cells, CTL, and intestinal intraepithelial lymphocytes. J Immunol. 1998;161(6): 2780-90.

34. Hua L, Lin H, Li D, Li L, Liu Z. Mining functional gene modules linked with rheumatoid arthritis using a SNP-SNP network. Genomics Proteomics Bioinformatics. 2012;10(1):23-34

35. Cai T, Li J, An X, Yan N, Li D, Jiang Y, et al. Polymorphisms in MIR499A and MIR125A gene are associated with autoimmune thyroid diseases. Mol Cell Endocrinol. 2017:440:106-15.

36. Song RH, Qin Q, Yan N, Muhali FS, Meng S, He ST, et al. Variants in IRAK1MECP2 region confer susceptibility to autoimmune thyroid diseases. Mol Cell Endocrinol. 2015:399:244-9.

37. Chen K, Zhou YX, Li K, Qi LX, Zhang QF, Wang MC, et al. A novel threeround multiplex PCR for SNP genotyping with next generation sequencing. Anal Bioanal Chem. 2016;408(16):4371-7.

38. Liu X, Yang S, Meng L, Chen C, Hui X, Jiang Y, et al. Association between PTCH1 and RAD54B single-nucleotide polymorphisms and non-syndromic orofacial clefts in a northern Chinese population. J Gene Med. 2018;20:e3055.

39. Steinberg MW, Cheung TC, Ware CF. The signaling networks of the herpesvirus entry mediator (TNFRSF14) in immune regulation. Immunol Rev. 2011;244(1):169-87.

40. Watanabe N, Gavrieli M, Sedy JR, Yang J, Fallarino F, Loftin SK, et al. BTLA is a lymphocyte inhibitory receptor with similarities to CTLA-4 and PD-1. Nat Immunol. 2003;4(7):670-9.

41. Sedy JR, Gavrieli M, Potter KG, Hurchla MA, Lindsley RC, Hildner K, et al. B and $\mathrm{T}$ lymphocyte attenuator regulates $\mathrm{T}$ cell activation through interaction with herpesvirus entry mediator. Nat Immunol. 2005:6(1):90-8.

42. Tao R, Wang L, Murphy KM, Fraser CC, Hancock WW. Regulatory T cell expression of herpesvirus entry mediator suppresses the function of $B$ and $T$ lymphocyte attenuator-positive effector T cells. J Immunol. 2008;180(10): 6649-55.

43. Galatola M, Cielo D, Panico C, Stellato P, Malamisura B, Carbone L, et al. Presymptomatic diagnosis of celiac disease in predisposed children: the role of gene expression profile. J Pediatr Gastroenterol Nutr. 2017;65(3):314-20.

44. Yang B, Huang Z, Feng W, Wei W, Zhang J, Liao Y, et al. The expression of BTLA was increased and the expression of HVEM and LIGHT were decreased in the T cells of patients with rheumatoid arthritis [corrected]. PLoS One. 2016:11(5):e0155345.

45. Ye Z, Deng B, Wang C, Zhang D, Kijlstra A, Yang P. Decreased B and T lymphocyte attenuator in Behcet's disease may trigger abnormal Th17 and Th1 immune responses. Sci Rep. 2016;6:20401.

46. Krause P, Zahner SP, Kim G, Shaikh RB, Steinberg MW, Kronenberg M. The tumor necrosis factor family member TNFSF14 (LIGHT) is required for resolution of intestinal inflammation in mice. Gastroenterology. 2014;146(7): 1752-62 e1754

47. Herro R, Shui JW, Zahner S, Sidler D, Kawakami Y, Kawakami T, et al. LIGHTHVEM signaling in keratinocytes controls development of dermatitis. J Exp Med. 2018;215:415

48. Perdigones N, Vigo AG, Lamas JR, Martinez A, Balsa A, Pascual-Salcedo D, et al. Evidence of epistasis between TNFRSF14 and TNFRSF6B polymorphisms in patients with rheumatoid arthritis. Arthritis Rheum. 2010; 62(3):705-10

49. Blanco-Kelly F, Alvarez-Lafuente R, Alcina A, Abad-Grau MM, de Las HV, Lucas $M$, et al. Members $6 B$ and 14 of the TNF receptor superfamily in multiple sclerosis predisposition. Genes Immun. 2011;12(2):145-8.

50. D'Addio F, Ueno T, Clarkson M, Zhu B, Vergani A, Freeman GJ, et al. CD160lg fusion protein targets a novel costimulatory pathway and prolongs allograft survival. PLoS One. 2013:8(4):e60391.

51. Wang Y, Subudhi SK, Anders RA, Lo J, Sun Y, Blink S, et al. The role of herpesvirus entry mediator as a negative regulator of $\mathrm{T}$ cell-mediated responses. J Clin Invest. 2005;115(3):711-7.

52. He W, Wang $B$, Li $Q$, Yao $Q$, Jia X, Song $R$, et al. Aberrant expressions of costimulatory and co-inhibitory molecules in autoimmune diseases. Front Immunol. 2019;10:261.

53. Ha SJ, West EE, Araki K, Smith KA, Ahmed R. Manipulating both the inhibitory and stimulatory immune system towards the success of therapeutic vaccination against chronic viral infections. Immunol Rev. 2008; 223:317-33.

54. Shukla SK, Singh G, Ahmad S, Pant P. Infections, genetic and environmental factors in pathogenesis of autoimmune thyroid diseases. Microb Pathog. 2018:116:279-88.

\section{Publisher's Note}

Springer Nature remains neutral with regard to jurisdictional claims in published maps and institutional affiliations.

Ready to submit your research? Choose BMC and benefit from:

- fast, convenient online submission

- thorough peer review by experienced researchers in your field

- rapid publication on acceptance

- support for research data, including large and complex data types

- gold Open Access which fosters wider collaboration and increased citations

- maximum visibility for your research: over $100 \mathrm{M}$ website views per year

At BMC, research is always in progress.

Learn more biomedcentral.com/submissions 